\title{
(5)
}

OPINION

\section{Improving care for patients with lung cancer in the UK}

\author{
Tariq Sethi, ${ }^{1}$ Eric Lim, ${ }^{2}$ Mick Peake, ${ }^{3}$ John Field, ${ }^{4}$ John White, ${ }^{5}$ Marianne Nicolson, ${ }^{6}$ \\ Corinne Faivre-Finn, ${ }^{7}$ Paul Cane, ${ }^{8}$ John Reynolds, ${ }^{9}$ Henrik Møller, ${ }^{10}$ Hilary Pinnock ${ }^{11}$
}

For numbered affiliations see end of article.

\section{Correspondence to}

Prof Tariq Sethi,

Head of Respiratory Medicine, King's Health Partners, London SE5 9RJ, UK; tariq.sethi@kcl.ac.uk

Received 27 September 2013 Revised 1 October 2013 Accepted 1 October 2013
To cite: Sethi T, Lim $\mathrm{E}_{\text {, }}$ Peake $\mathrm{M}$, et al. Thorax 2013;68:1181-1185.

\section{INTRODUCTION}

The care of patients with lung cancer in the UK today has improved over the last 10 years, primarily because we are now able to measure and quantify the differences in patient care pathways and outcomes. The National Lung Cancer Audit started in 2004 with $40 \%$ case ascertainment and today it is inconceivable that any NHS hospital that manages patients with lung cancer would not contribute. With $100 \%$ case ascertainment since $2010,{ }^{1}$ active treatment rates increased from $45 \%$ in 2005 to $61 \%$ in 2012 and the overall surgical resection rates rose from $9 \%$ to $16 \%$ in the same period. Measurements of performance and outcome data can drive improved survival in patients with lung cancer, and the establishment of national data collection is an important step towards improving the quality of patient care. ${ }^{2}$

However, even with these improvements, survival from lung cancer in the UK lags behind that of comparable countries. ${ }^{3}{ }^{4}$ The reasons for this in part are due to late diagnosis and huge variations in the delivery of care across the country. A round table meeting assembled experts with the remit to explore what changes were needed in the current management of patients with lung cancer in the UK to improve their survival.

To provide a snapshot of how respiratory physicians view the current state of lung cancer management in the UK, Thorax sent out an online survey in August 2013 to 6000 hospital physicians identified as having an interest in respiratory medicine. This group was chosen as respiratory physicians are the 'gatekeepers' who determine entry into lung cancer diagnostic and care pathways: their attitudes have great influence on lung cancer patients. Responses were received from $3-4 \%$ of the survey group, who are likely to represent a particularly motivated group of respiratory physicians with an interest in lung cancer.

Respondents thought that there would have been opportunities to make a diagnosis significantly earlier, on average, for about one-third of patients, and two-thirds of physicians believed that greater public awareness of the symptoms of lung cancer is likely to have a significant impact on the outcome for patients.

Some findings from the survey were surprising. For example, three-quarters of respondents did not think that lung cancer should be managed in fewer centres than at present despite the evidence of significantly better lung cancer survival in large centres. ${ }^{6}$ However, $85 \%$ of respondents supported the policy that patients with early stage disease who are turned down for surgery should be offered a second opinion. Sixty-seven per cent of respondents believed that stage IIIB/IV non-small cell lung cancer is worth treating in most patients-something that is perhaps not borne out in practice.

When asked about the factors that contribute to the poor survival rate from lung cancer in the UK, the respondents ranked low resection rates and slow uptake of new advances in treatment as the main reasons; lower use of radiotherapy, lack of screening, poor research funding and lower lung cancer drug use ranked well below the first two cited reasons.

\section{EPIDEMIOLOGY}

The epidemiology of lung cancer is a good starting point to drive improvements in the management of lung cancer. Examination of UK survival rates shows that the death rate from lung cancer is significantly higher in all age groups compared with Norway and Sweden, but that this difference is marked only in the first 3 months of follow-up which suggests later presentation with more advanced disease in the UK. ${ }^{4}$ In addition, there are disparities in stage-specific survival, suggesting that other factors are also important such as differences in treatment. Epidemiological research also identified that survival from lung cancer improves with increasing use of surgical resection and also with stereotactic ablative body radiotherapy (SABR)..$^{5} 7$ However, resection rates in England remain low compared with other developed countries, and vary more than threefold between hospital trusts from a low of $4 \%$ to a high of $15 \%{ }^{8}$ This has been attributed in part to the availability of specialist surgeons and their willingness to operate. Although resection rates are currently increasing in the UK, it is true to say that we are still a long way away from the point where increasing resection rates will do more harm than good. While there is a general call for greater surgical intervention in patients with lung cancer, there is also a need for caution as the results from surgical units with low volumes of patients $(<70$ per year) are associated with worse outcomes than those with higher throughputs. ${ }^{6}$ Better short-term survival observed in busier surgical units is likely to reflect greater skill, experience, specialisation and infrastructure, while improved long-term survival possibly results from better staging and management of patients.

\section{SCREENING}

One of the major questions in lung cancer management is whether screening would lead to better 
survival. The National Lung Cancer Screening Trial from the USA published in 2011 suggested a 20\% improvement in lung cancer survival in patients aged $>55$ years who have smoked $>30$ pack years who were randomised to low-dose CT screening. ${ }^{9}$ The Dutch-Belgian randomised lung cancer screening trial (NELSON) to investigate whether CT screening will reduce mortality in high-risk individuals is due to report in $2015 .{ }^{10}$ However, discussion should begin now on the frequency of screening, the target age group and the cost implications of a UK screening programme. Another emerging area of importance and concern is the identification of risk factors and screening of lifelong non-smokers who now make up $10-15 \%$ of the lung cancer population.

\section{PRIMARY CARE}

While discussions on screening are ongoing, there are some relatively straightforward ways to improve lung cancer care and survival. Evidence suggests that screening for early symptoms of lung cancer can also improve survival. Increased public awareness of indicative symptoms, prompt referral by GPs of suspected cases, easy and quick access to x-ray services and a coordinated pathway to CT scanning, respiratory physicians and then the multidisciplinary team (MDT) are all essential. The primary care practitioner has a central role in the identification and referral of patients with suspected disease and the prevention of lung cancer through smoking cessation strategies. However, many questions remain-specifically around what cardinal symptoms the public should be made aware of to prompt a visit to the GP. The standard primary care investigation of a chest $\mathrm{x}$-ray misses up to one-quarter of cases of lung cancer, ${ }^{8}$ and direct timely access to CT scanning may be a better approach to aid earlier diagnosis. However, this has clear resource implications in terms of personnel and machines as well as finance.

\section{RADIOLOGY}

One of the stumbling blocks to achieving a rapid diagnosis is the shortage of radiologists in the UK. For every 100000 people in the UK there are currently 4.6 radiologists compared with a European average of 9-10/100 000 and 18/100 000 in Denmark. The College of Radiologists has acknowledged the detrimental impact of the UK's provision of radiologists and has recommended increasing the number of trainees to address the shortfall. ${ }^{13}$ However, because training takes 5 years, it will be some time before the number of radiologists is likely to increase. Even then, and especially with the squeeze on funding, the UK may never be served by radiologists to the same extent as other comparable countries. Alternative solutions include the adoption of advanced radiographer reporting. Evidence has indicated that appropriately trained radiographers can report chest $\mathrm{x}$-rays and CT scans with comparable accuracy to radiologists. ${ }^{14}$ While contentious, given the current strain on services, staffing and equipment, the advancement of radiographers presents a realistic way of increasing the capacity of radiological services and reducing delays in diagnosis.

\section{PATHOLOGY}

Advances in systemic therapies underscore the importance of the acquisition of good quality tissue samples in patients with lung cancer. The current histological confirmation rate of $76 \%$ means that one-quarter of patients are unable to receive any tailored therapy and there may be scope for improvement. Tissue from lung cancers may be difficult to obtain and it may be necessary to determine the histological type, subtype and measurement of several molecular targets, often from small samples. In order to obtain the best quality samples possible and to perform effective histological assessment, specialisation of both the clinicians obtaining the samples and the pathologists carrying out the test is desirable. Many hospitals are now setting up an endobronchial ultrasound service (EBUS); the lack of adequate training, experience and quality assurance will negatively impact on patient care and survival and, in addition, evidence suggests will be wasteful of valuable resources. To get the best outcomes, EBUS services should be monitored and accredited to meet nationally accepted standards. Insufficient specialisation of pathologists in many centres where lung cancers are diagnosed means that the quality of reporting can be variable. One solution is to encourage pathologists to participate in a national quality assurance scheme and, as personalised (stratified) medicine becomes more widespread, the need for efficient pathways for molecular testing of biomarkers such as epidermal growth factor receptor mutations is imperative.

\section{THORACIC SURGERY}

The patient should be the focus of our care, and improving patient care can be a focus for change. In terms of surgery for lung cancer, this means restricting operations to high-volume centres undertaken by specialist thoracic surgeons. There is now increasing but not universal availability of keyhole surgery (video-assisted thoracic surgery) in specialist centres in the UK. The indications for surgery could be broadened by simply changing the attitude of referring clinicians and patients to consider surgery as an equal option when the outcomes appear equal (eg, in single station N2 disease) and where there has not been any evidence supporting benefit in overall survival between surgery as part of multimodality management against medical treatment alone. ${ }^{15}$ To definitively prove this, a randomised controlled trial (RCT) using modern radiotherapy and surgical techniques is required, highlighting the urgent need for RCTs to define optimal lung cancer treatment pathways. Including patients in the decision-making process and taking into account their opinion of what constitutes 'high risk' has been shown to improve compliance and overall satisfaction of care. ${ }^{16}$

\section{MEDICAL ONCOLOGY}

Another factor holding back progress in the UK is the struggle to improve the rate of adjuvant treatment. The current TNM classification system may not effectively capture patients who remain in the high-risk categories despite early cancer stage, so we need to embrace new technologies such as circulating tumour cells and better pathological descriptions of such things as the level of vascular invasion. With the ever increasing evidence of the efficacy of targeted treatments, many of which have a better toxicity profile than standard chemotherapy, mutation testing needs to be universal and probably means that greater efforts need to be made to obtain tissue for a morphological and molecular diagnosis. For many patients with lung cancer, the outlook is more positive than ever before. It should rarely be the case that a patient is turned down for treatment without a biopsy. As more new drug treatments for lung cancer become available, there is an increasing problem of finding the funds to pay for those that have proved to be effective, especially those that have not been approved by the National Institute for Health and Care Excellence (NICE) or the Scottish Medicines Consortium (SMC). However, the main outcome of overall survival that is used by NICE as the basis for their evaluation of cost-effectiveness is not easily applicable to most new targeted agents where crossover to other treatments occurs after 
patients have received the trial drug first line. There needs to be a distinction between patient-centred and 'value'-centred healthcare, but it is not at all clear how this can be done. Not funding the drugs proved to improve outcomes and survival raises important ethical considerations and is a difficult concept to grasp by patients who have taken part in trials in the belief that they are doing so to advance the care of patients who will follow them.

\section{CLINICAL ONCOLOGY}

Advances in radiotherapy mean than many patients with lung cancer who could not be treated in the past can now be treated radically. This requires the widespread implementation of modern radiotherapy technology such as intensity modulated radiotherapy, SABR and image-guided radiotherapy which can deliver highly focused radiotherapy to the tumour and not the surrounding tissues. Access to these techniques is limited more by resource limitations at the level of physicists and therapy radiographers than the machinery itself. It also requires more patients to be referred for treatment in accordance with national and international guidelines, ${ }^{15}$ where adherence and a reduction in the variation in practice have the potential to improve the quality of care. With the ever more complex range of radiotherapy techniques, radiation oncology itself probably needs to be further developed as a subspecialty.

\section{LUNG CANCER NURSE SPECIALISTS}

Another area that can be improved is access to clinical trials in all treating centres, including groups of patients who are often excluded from research such as older patients and those with comorbidities. In addition, the ongoing collection and reporting of data on what happens to all of our patients-and not just those who enter clinical trials-is essential. There remains an overwhelming importance to focus on the well-being of patients in their entire pathway. Lung cancer specialist nurses (LCNs) fulfil a vital role in the MDT and are probably the people who patients will see most in their treatment journey. LCNs should be involved for the entire patient pathway from pre-diagnosis to treatment thereafter, and the LCN should be recognised as the patient's advocate in the MDT. LCNs are in an ideal position to be able to provide care in a holistic way, ensuring that all the patient's care needs are addressed, assessing physical, psychological, social, spiritual and financial needs, provision of information, symptom management interventions (including prescribing), care coordination and health promotion. Follow-up by LCNs should be offered to all patients. The National Lung Cancer Audit report suggests that patients who are known to a LCN are twice as likely to receive active treatment $(60 \%$ vs $30 \%)$ than those patients who are not. This observation is likely to be multifactorial (eg, good MDT functioning), but is suggestive of the importance of the role of the LCN in terms of treatment uptake. ${ }^{17}{ }^{18}$ For the future it is essential that LCNs are supported with evidence generated for policy makers, commissioners, providers and clinicians to facilitate promotion of their role. Early involvement of palliative care and attention to the nutritional state and encouragement of exercise/rehabilitation can improve both morbidity and survival. The improved survival may also reflect the importance of holistic assessment and management of general health problems in people who often have multiple comorbidities. ${ }^{19}$

\section{SMOKING CESSATION}

Efforts to support smoking cessation have yielded very encouraging results in the UK, and the evidence strongly supports the use of nicotine replacement therapy or pharmacological agents such as bupropion or varenicline to aid smoking cessation. E-cigarettes are still unlicensed and therefore cannot be recommended until their long-term safety has been determined. Furthermore, while e-cigarettes were initially viewed as a nicotine substitute similar to patches and chewing gum, campaigns are now emerging where companies are promoting e-cigarettes in the same way as tobacco firms once did to sell cigarettes-as a lifestyle choice to people who have never smoked. There is a grave concern that non-smokers are being attracted into this new market, with advertising campaigns that may lead to a rise in cigarette smoking. Any moves in this regard must be opposed in the strongest possible terms.

The research base for lung cancer in the UK needs urgent strengthening, further to that seen in recent months. Lung cancer still attracts the least funding of all common tumours yet has the lowest survival rates. ${ }^{20}$ Ring-fenced funding for lung cancer is required, since such funding will inevitably lead to increased research and increase the base for training and expertise, developing doctors with a specific interest in lung cancer. In addition, research will help further understanding of the basic biology to allow stratification of patients to more personalised treatments through identification of biomarkers. Identifying those at highest risk will improve early diagnosis and treatment outcomes. There are a number of critical clinical decision points, such as N2 disease, for which there is no clear evidence regarding the best treatment. Research should also focus on interventions to improve the general health of patients after radical treatment. More clinical trials are required, and the portfolio will be strengthened by allowing clinicians more time to undertake this type of research activity. We recommend allocation of extra research programmed activity payments to qualified interested lung cancer specialist clinicians to fulfil this purpose.

\section{ACTION POINTS}

The panel agreed the following action points would make an immediate and significant impact on the mortality, morbidity and survival of patients suffering from lung cancer:

1. Improve smoking cessation.

- Mandatory recording of smoking status and provision of smoking cessation advice to patients who smoke on admission notes to hospital (as has already been incentivised in primary care ${ }^{21}$ ), with provision of brief advice and the offer of referral to further support for those who smoke.

- Offer nicotine replacement therapy or bupropium or varenicline in conjunction with psychological support for all those willing to stop smoking.

- Await NICE guidance for e-cigarettes, particularly as they may promote smoking behaviour.

2. Earlier diagnosis

- Evidence suggests that early diagnosis of symptomatic lung cancer can improve survival.

- Realistic publicity about symptoms should encourage awareness among patients.

- Radiology departments to work towards making a CT scan, rather than chest $\mathrm{x}$-ray the initial investigation for suspected lung cancer (including in primary care) and timely access to diagnostic facilities. Advanced practitioner radiographers should be trained to report $\mathrm{x}$-rays/ CT scans.

- Discussion should begin now on the frequency of screening, the target age group and cost implications of a UK 
screening programme using CT scans or other validated screening methods. Another emerging area of importance and concern is the identification of risk factors and screening of lifelong non-smokers who now make up 10$15 \%$ of the lung cancer population.

- Care pathway: improve access to rapid diagnostic facilities to allow all patients with suspected lung cancer to undergo a CT scan as the first-line investigation.

3. Endobronchial ultrasound (EBUS)

- EBUS services should be properly regulated, available in large centres with significant experience and good outcomes in terms of sensitivity and specificity of diagnosis accuracy.

- These centres should be nationally accredited by the British Thoracic Society.

4. Multidisciplinary team (MDT)

- Evidence suggests that large centres with dedicated consultant specialists with a specific interest in lung cancer have the best outcomes in terms of survival. For example, high-volume surgical centres have better outcomes despite operating on older, less fit patients. ${ }^{6}$

- Large specialist centres have the highest resection rates and chemotherapy rates. MDTs should consist of specialist consultant teams from respiratory medicine, palliative care, thoracic surgery, clinical and radiation oncology, pathology and cancer clinical nurse specialists with a specific interest in lung cancer.

- Early involvement of palliative care in the patient's treatment will improve quality of life and may improve survival.

- Patients with lung cancer often have multiple comorbidities, the treatment of which may improve quality of life and survival and needs a holistic approach.

- Patients should get specialist advice for their treatment options and their outcomes, allowing them to make more informed decisions about their care.

- Continue to promote and expand the pool of cancer clinical nurse specialists who should be involved in the entire patient journey and recognised as the patient's advocate in the MDT.

5. Pathological diagnosis

- Pathological confirmation of lung cancer should be undertaken in all patients unless they are too unwell for tyrosine kinase inhibitor therapy or investigation would be life-threatening.

- Pathologists involved with an MDT should take part in a specialist lung cancer quality assurance scheme to be developed through the Royal College.

- Efficient pathways for testing biomarkers such as epidermal growth factor receptor should be present in all lung cancer centres.

6. Research

- Lung cancer is the lowest funded of all the malignancies. The British Thoracic Society should lobby government and Cancer Research UK for ring-fenced support for lung cancer.

- Consultants with a specialist interest in lung cancer should be awarded research programmed activity payments to encourage participation in clinical trials to improve the clinical trial base for lung cancer.

- Highlight that overall survival is not always an appropriate end point in trials for the assessment of costeffectiveness of many new targeted agents in lung cancer; progression-free survival should be accepted by NICE and SMC as satisfactory evidence of improved therapeutic outcome for some trials However, short-term allcause mortality is a sensitive measure for a number of trials and other studies.

\section{Author affiliations}

${ }^{1}$ Department of Respiratory Medicine and Allergy, King's Health Partners, London, UK

${ }^{2}$ Department of Thoracic Surgery, Royal Brompton Hospital, National Heart and Lung Institute, Imperial College, London, UK

${ }^{3}$ Department of Respiratory Medicine, Glenfield Hospital, National Clinical Lead for Lung Cancer, Leicester, UK

${ }^{4}$ Roy Castle Lung Cancer Research Programme, University of Liverpool Cancer Research Centre, Liverpool, UK

${ }^{5}$ Leeds Teaching Hospital NHS Trust and Chair, National Lung Cancer Forum for Nurses, Leeds, UK

${ }^{6}$ Department of Medical Oncology, Aberdeen Royal Infirmary, Aberdeen, UK ${ }^{7}$ Department of Clinical Oncology, Christie Hospital, Manchester, UK

${ }^{8}$ Department of Pathology, Guy's and St Thomas' NHS Foundation Trust, London, UK

${ }^{9}$ Department of Radiology, Birmingham Heartlands Hospital, Birmingham, UK ${ }^{10}$ Department of Epidemiology, King's Health Partners Integrated Cancer Centre, Guy's Hospital, London, UK

${ }^{11}$ Allergy and Respiratory Research Group, Centre for Population Health Sciences, University of Edinburgh and Principal in General Practice, Whitstable, UK

Contributors A round table discussion hosted by Thorax. Organised and summary edited by Eric Lim and Tariq Sethi.

Chair Dr Mick Peake, Consultant Respiratory Physician, Glenfield Hospital, Leicester, and National Clinical Lead for Lung Cancer.

Respiratory Medicine: Professor Tariq Sethi, Head of Respiratory Medicine, King's Health Partners, London.

Thoracic Surgery: Mr Eric Lim, Consultant Thoracic Surgeon, Royal Brompton Hospital and Senior Lecturer, National Heart and Lung Institute, Imperial College, London.

CT Screening: Professor John Field, Liverpool, Clinical Chair in Molecular Oncology, University of Liverpool and Director of Research of the Roy Castle Lung Cancer Research Programme, The University of Liverpool Cancer Research Centre, Department of Molecular and Clinical Cancer Medicine, Liverpool.

Nurse specialist: Mr John White, Lead Macmillan Lung Cancer Nurse Specialist, Leeds Teaching Hospital NHS Trust and Chair, National Lung Cancer Forum for Nurses.

Medical Oncology: Dr Marianne Nicolson, Consultant Medical Oncologist, Aberdeen Royal Infirmary.

Clinical Oncology: Dr Corinne Faivre-Finn, Consultant Clinical Oncologist, Christie Hospital, Manchester.

Pathology: Dr Paul Cane, Consultant Thoracic Pathologist and Thoracic MDT Lead, Guy's and St Thomas' NHS Foundation Trust, London.

Radiology: Dr John Reynolds, Consultant Radiologist, Birmingham Heartlands Hospital.

Epidemiology: Professor Henrik Møller, Professor of Cancer Epidemiology and Population Health, King's Health Partners Cancer Centre, Guy's Hospital, London.

GP: Dr Hilary Pinnock, Reader, Allergy and Respiratory Research Group, Centre for Population Health Sciences, University of Edinburgh and Principal in General Practice, Whitstable.

Funding This initiative is sponsored by Eli Lilly and Company Limited through the provision of an unrestricted educational grant. Lilly has had no influence over the content other than a review for medical accuracy.

Competing interests $\mathrm{PC}$ has received speaker fees from Lilly, Roche and BL. MN has received financial support from Lilly, Roche, Boehringer Ingelheim, Amgen, Novartis, BMS, Ostika and GSK for advisory board work, lectures and meetings and is the Director of Lung Cancer Screening at the Cromwell Hospital. EL has received travel sponsorship from Coviclen and Stapler Company. JF, TS, CF-F, HM, JR, HP, JW have no competing interests.

Provenance and peer review Not commissioned; internally peer reviewed.

\section{REFERENCES}

1 National Lung Cancer Audit. Report for the audited period 2010. The Information Centre, National Lung Cancer Audit, 2011. 
2 Lim E, Popat S. What exactly are we doing to improve low lung cancer survival in the united kingdom? Thorax 2013;68:504-5.

3 Walters S, Maringe C, Coleman MP, et al. Lung cancer survival and stage at diagnosis in Australia, Canada, Denmark, Norway, Sweden and the UK: a population-based study, 2004-2007. Thorax 2013:68:551-64.

4 Holmberg L, Sandin F, Bray F, et al. National comparisons of lung cancer survival in England, Norway and Sweden 2001-2004: differences occur early in follow-up. Thorax 2010;65:436-41.

5 Waller DA. Whose risk is it anyway? Thorax 2013;68:799-800.

6 Luchtenborg M, Riaz SP, Coupland VH, et al. High procedure volume is strongly associated with improved survival after lung cancer surgery. J Clin Oncol 2013:31:3141-6.

7 Palma D, Visser O, Lagerwaard FJ, et al. Impact of introducing stereotactic lung radiotherapy for elderly patients with stage I non-small-cell lung cancer: a population-based time-trend analysis. J Clin Oncol 2010;28:5153-9.

8 Riaz SP, Luchtenborg M, Jack RH, et al. Variation in surgical resection for lung cancer in relation to survival: Population-based study in England 2004-2006. Eur J Cancer 2012;48:54-60.

9 National Lung Screening Trial Research Team, Aberle DR, Adams AM, et al. Reduced lung-cancer mortality with low-dose computed tomographic screening. New Eng J Med 2011;365:395-409.

10 Field J, van Klaveren R, Pedersen J, et al. European randomised lung cancer screening trials: Post NLST. J Surg Oncol 2013;108:280-6.

11 Field J, Oudkerk M, Pedersen J, et al. Prospects for population screening and diagnosis of lung cancer. Lancet 2013;382:732-41.
12 Hamilton W, Peters TJ, Round A, et al. What are the clinical features of lung cancer before the diagnosis is made? A population based casecontrol study. Thorax 2005;60:1059-65.

13 Royal College of Radiologists. Investing in the clinical radiology workforce - the quality and efficiency case. London, June 2012. http://www.rcr.ac.uk/docs/radiology/ pdf/RCR CRWorkforce_June2012.pdf

14 Brealey S, Scally A, Hahn S, et al. Accuracy of radiographer plain radiograph reporting in clinical practice: A meta-analysis. Clinical Radiology 2005;60:232-41.

15 Lim E, Baldwin D, Beckles M, et al. Guidelines on the radical management of patients with lung cancer. Thorax 2010;65(Suppl 3):iii1-27.

16 Lim E. Patients' perspective in the surgical decision-making process. Thorac Surg Clin 2012;22:539-43.

17 Roy Castle Lung Cancer Foundation. Understanding the value of lung cancer nurse specialists. http://www.roycastle.org/news-and-campaigning/Campaigns/ Improving-lung-cancer-treatment-and-care/ Understanding-The-Value-of-Lung-Cancer-Nurses

18 National Lung Cancer Forum for Nurses and the Roy Castle Lung Cancer Foundation (2013). Understanding the value of lung cancer nurse specialists . http:// www.roycastle.org/Resources/Roy 20Castle/Documents/PDF/UnderstandTheValueO flungCancerNurseSpecialists_V03.pdf

19 Temel JS, Greer JA, Muzikansky A, et al. Early palliative care for patients with metastatic non-small-cell lung cancer. N Engl J Med 2010;363:733-42.

20 BBC News - How under-funded is lung cancer research? www.bbc.co.uk/news/ business-22310825

21 NHS Confederation, British Medical Association. New GMS Contract 2003: investing in general practice. London, March 2003. 